\title{
Rayleigh Region in Amorphous and Nanocrystaline FINEMET Alloy
}

\author{
L. NOVÁK ${ }^{a, *}$, J. KOVÁC̆ ${ }^{b}$ \\ ${ }^{a}$ Department of Physics, Faculty of Electrical Engineering and Informatics, Technical University of Kosice, Letná 9, \\ 04200 Košice, Slovakia \\ ${ }^{b}$ Institute of Exp. Physics, Slovak Academy of Sciences, Watsonova 47, 04001 Košice, Slovakia
}

\begin{abstract}
The magnetization processes in region of small excitation magnetic fields - Rayleigh region, were investigated on the amorphous and nanocrystalline FINEMET ribbons. The significant differences in behaviour of these materials were found. This could be explained by the difference in the value of magnetoelastic anisotropy as well as by the different thickness of the domain walls in these materials.
\end{abstract}

DOI: 10.12693/APhysPolA.126.126

PACS: 75.50.Kj, 75.50.Tt, 75.60.Ch, 75.60.Ej, 75.60.Jk

\section{Introduction}

Magnetization processes in ferromagnetic materials can be described in four ways, reversible and irreversible domain wall (DW) motion, rotation of the vector of magnetic polarization, and paraprocess in high magnetic fields. The process of reversible DW motion is characteristic for the range of small exciting magnetic fields - the Rayleigh region ( $R R$ ).

The dependence of magnetic polarization $J$ in this region, called also the region of initial susceptibility (permeability), on the magnetic field strength $H$ can be expressed by the following equation

$$
J=\kappa_{p}\left(\mu_{0} H\right)+\alpha\left(\mu_{0} H\right)^{2}
$$

where $J$ is magnetic polarization, $H$ is magnetic field strength, $\kappa_{p}$ is initial volume magnetic susceptibility, $\alpha$ is Rayleigh's constant and $\mu_{0}$ is permeability of vacuum.

The initial volume magnetic susceptibility and Rayleigh constant are parameters depending on the properties of ferromagnetic materials [1].

The irreversible DW motion at the end of RR is caused by the presence of defects in the atomic structure or nonferromagnetic regions. The occurrence of these regions, the inclusions, in the DW decreases wall energy, creating potential well which anchors the DW in the position of the inclusion. For simplicity we assume the inclusion of spherical shape. The maximum of energy to move the DW is needed in the case when the thickness of a DW $\left(\delta_{w}\right)$ is approximately the same as the diameter of the inclusion $(d)$ present in the wall. In RR there are also some DWs anchored due to the presence of defects. The magnetization process then takes place by reversible motion of the DWs which are not held by inclusions, since very small magnetic fields, characteristic for this region, cannot overcome the pinning of the wall by the inclusion.

*corresponding author; e-mail: ladislav.novak@tuke.sk
There is a question about the extent to which the RR can be influenced by the structure of material, amorphous or crystalline, and by various magnetic properties, resulting from different atomic structures of particular materials.

\section{Experimental}

Two samples were chosen for the study of this problem: FINEMET in as-quench amorphous state and thermally treated $\left(520^{\circ} \mathrm{C}, 1\right.$ hour $)$ nanocrystalline FINEMET.

The magnetic parameters of the samples were determined by a computer-controlled magnetometer using two fluxgate-type probes for measuring the stray field of the sample. The curves of initial magnetic polarization were measured in detail for both samples after careful demagnetization performed in alternating field with decreasing amplitude. Subsequently, the full hysteresis loops were also measured. The other parameters, such as coercivity, value of magnetoelastic anisotropy, etc. were estimated from these data. The selected magnetic parameters of the studied samples, saturated magnetic polarization $\left(J_{s}\right)$, measured in excitation field of the $15 \mathrm{kA} / \mathrm{m}$, coercivity $\left(H_{C}\right)$, magnetoelastic anisotropy $\left(K_{\sigma}\right)$, determined from area over the descending branch of hysteresis loop [3] and Curie temperature $\left(T_{C}\right)$ are listed in Table I.

TABLE I

The selected magnetic parameters of the samples.

\begin{tabular}{c|c|c|c|c}
\hline \hline FINEMET & $J_{s}(\mathrm{~T})$ & $H_{C}(\mathrm{~A} / \mathrm{m})$ & $K_{\sigma}\left(\mathrm{Jm}^{-3}\right)$ & $T_{C}(\mathrm{~K})$ \\
\hline amorph & 1.159 & 17.1 & 839 & 600 \\
\hline nanocryst. & 1.164 & 2.04 & 465 & 860
\end{tabular}

\section{Results and discussion}

The measured points of the beginning parts of the curves of initial magnetic polarization were fitted to the equation (1). The fitting parameters make it possible to calculate $\kappa_{p}$ and $\alpha$. These parameters are listed in Table II. 
Figure 1 displays the curves of initial magnetic polarization for both samples together with the Rayleigh parabolas. For the amorphous sample the Rayleigh parabola, obeying the equation (1), coincides with the measured data in the range from 0 up to around $8 \mathrm{~A} / \mathrm{m}$, for the nanocrystalline sample this interval is up to $10.5 \mathrm{~A} / \mathrm{m}$ (marked by arrows in the figure). This relatively small difference is unexpected, considering a big difference in coercivity.

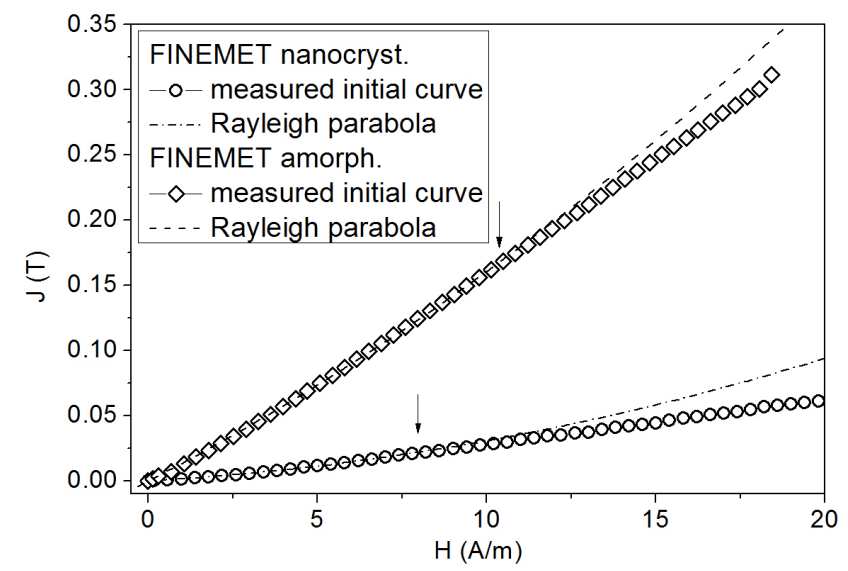

Fig. 1. The curve of initial magnetic polarization and Rayleigh parabola of the amorphous and nanocrystalline FINEMET.

The significant difference is in the degree of the magnetization of sample in the range of RR. If we consider that the magnetized part is the ratio of the maximum value of magnetic polarization achieved in $\mathrm{RR}$ to the saturated magnetic polarization (in the magnetic field of $15 \mathrm{kA} / \mathrm{m}$ ), we can assume that $2.1 \%$ of the volume is magnetized in the amorphous sample and $14.7 \%$ in the nanocrystalline one.

From the presented results it follows that the mobility of DWs in RR is several times lower for amorphous sample in comparison with nanocrystalline one. This fact can by caused by the difference in atomic structure as well as by the difference in the thickness of the DW.

We attempt to estimate the thickness of $180^{\circ}-\mathrm{DW}$ as well as the energy needed for the creation of unit area of such wall for both samples. For calculation of the thickness of $180^{\circ}$-DW one can use the formula [2]:

$$
\delta_{\omega}=\sqrt{\frac{A S^{2} \psi^{2}}{K a}},
$$

where $S$ is value of orientation of spins, for ferromagnetic material we take $S=1, A$ is exchange integral, $\psi$ is the angle between the directions of spontaneous magnetization in neighbouring domains, in the case of $180^{\circ}$ wall it is $\psi=\pi, K$ is the value of the unit-volume energy of the total magnetic anisotropy. For an amorphous ferromagnetic material we can write $K_{a m}=K_{\sigma}=839 \mathrm{~J} \cdot \mathrm{m}^{-3}$. For nannocrystalline FINEMET according to random anisotropy model [4] the magnetocrystalline anisotropy is suppressed, so in this case we can also assume $K_{\text {nano }}=$ $K_{\sigma}=465 \mathrm{~J} \cdot \mathrm{m}^{-3}$. $a$ is the grid spacing, for FeSi alloy with low content $\mathrm{Si}$ its value is $a=2.86 \times 10^{-10} \mathrm{~m}$. We have used this parameter in respect of composition of samples. Taking into account the above-mentioned considerations, the formula (2) can be rearranged:

$$
\delta_{\omega}=\pi \sqrt{\frac{A}{K a}} \sqrt{\frac{0.15 k T_{C}}{K a}},
$$

where $k$ is Boltzmann constant [2]. The thickness of $180^{\circ}$-DW was calculated using equation (3) for both samples and the obtained values are listed in Table II. In Table II are listed also $\kappa_{p}, \alpha$, relative degree of magnetization of sample $J_{\text {Rayl }} / J_{s}$, and thickness of DW $\delta_{\omega}$. Our values of $\kappa_{p}$ and $\alpha$ for amorphous material are in good agreement with the results of Kronmüller [5]

TABLE II

The parameters calculated from measured data.

\begin{tabular}{c|c|c|c|c}
\hline \hline FINEMET & $\kappa_{p} \cdot 10^{-3}$ & $\alpha\left(\mathrm{T}^{-1}\right)$ & $J_{\text {Rayl }} / J_{s}(\%)$ & $\delta_{\omega}(\mathrm{nm})$ \\
\hline amorph. & 1.16 & $103.10^{6}$ & 2.1 & 185 \\
\hline nanocryst. & 10.61 & $174.10^{6}$ & 14.7 & 296
\end{tabular}

\section{Conclusions}

In case of amorphous sample the portion of remagnetized volume in RR is small, thus the movement of DW is also small. This is in agreement with relatively high coercivity. The reason for this is in high internal stresses introduced into material during preparation, which causes high magnetoelastic anisotropy. Another reason can be also the clusterization of atoms, taking into account multicomponent nature of alloy. The thickness of DW, which is close to dimension of barriers, also contributes to this effect.

In case of nanocrystalline sample the scale of $R R$ is comparable with amorphous one, the portion of remagnetized volume in this region is significantly higher, which suggests increasing of the mobility of DW. This suggestion is also in agreement with a very low value of coercivity in comparison with amorphous sample. This effect is probably caused by processes of nannocrystalline transformation, where the relief of internal stresses occurs, which decreases the magnetoelastic anisotropy and the number of regions with braked DW movement. Simultaneously the thickness of DW, which is much higher than the dimensions of nanocrystallites $(10 \mathrm{~nm})$, increases.

\section{Acknowledgments}

This work was supported by Slovakian projects VEGA $1 / 0148 / 12$ and $1 / 0861 / 12$.

\section{References}

[1] J.M.D. Coey, Magnetism and Magnetic Materials, Oxford University Press, 2009.

[2] S. Chikazumi, Physics of Ferromagnetism, Oxford University Press, 1997.

[3] M. Koval'aková, P. Kollár, L. Novák, L. Ševcovič, J. Magn. Magm. Mater. 304, e648 (2006).

[4] G. Herzer, IEEE Trans. Mag. 26, 1397 (1990).

[5] H. Kronmüller, M. Fähnle, M. Domann, H. Grimm, R. Grimm, B. Gröger, J. Magn. Magm. Mater. 13, 53 (1979). 